\title{
KLEINER MOTOR, KLEINER MARKT
}

Wir

\section{entwickeln, was bewegt}

Liebe Leserin, lieber Leser,

mit dem BMW i3 geht aktuell das erste Elektrofahrzeug in Serie - zumindest bezogen auf die serielle Einbindung des Verbrennungsmotors -, das optional mit einem Range Extender ausgerüstet werden kann. Dabei ist die Stückzahlerwartung des Münchner Herstellers selbst nicht sonderlich hoch: Von den jährlich 40.000 Kunden für das Elektrofahrzeug werden weltweit nur $10 \%$, also 4000, den Reichweitenverlängerer ordern, schätzen Experten von BMW. Bezogen auf einen durchschnittlichen Modellzyklus von sieben Jahren werden es somit nicht einmal 30.000 Range-Extender-Aggregate sein, die BMW für den i3 in Summe herstellen wird. Da kann man sich schon fragen, ob der Aufwand für Entwicklung und Applikation des Range Extenders für diese kleine Stückzahl lohnt. Skaleneffekte in der Fertigung sind bei solchen Miniserien kaum zu erwarten. BMW hat zumindest den Vorteil, auf einen hauseigenen Zweizylinder-Motorradmotor als Basis zurückgreifen zu können. Dennoch verlangt das Unternehmen für den Range Extender einen Aufpreis von rund 4500 Euro - vergleichsweise viel, wenn man den Nutzen für den Endkunden gegenrechnet. Dazu kommt, neben zusätzlichem Gewicht und Wartungsaufwand, der Platzbedarf des Aggregats.

Auch insgesamt wird der Gesamtmarkt für Range-Extender-Aggregate künftig überschaubar bleiben: Sollten im Jahr 2020 wirklich weltweit 2 Millionen Elektroautos im Markt sein, was ohnehin fraglich ist, werden davon allerhöchstens 200.000 Fahrzeuge mit seriellem Range Extender ausgerüstet sein, vermutlich sehr viel weniger. Diese Gesamtzahl teilt sich dann noch auf unterschiedliche Regionen, verschiedene technische Lösungen und natürlich die einzelnen Marktplayer auf. Als weiterer marktlimitierender Faktor kommt die fortschreitende Batterieentwicklung hinzu: Steigende Leistungsfähigkeit und stetig sinkende Kosten der Energiespeicher könnten ab 2020 dazu führen, dass der Range Extender im Kosten-Nutzen-Vergleich weiter an Boden verliert. Die größte Konkurrenz für den Elektroantrieb mit Range Extender ist allerdings der Plug-in-Hybrid, der auch lokal emissionsfreies Fahren und sogar eine bessere Langstreckentauglichkeit bietet. Er befriedigt das Mobilitätsbedürfnis der breiten Käuferschicht und wird entsprechende Stückzahlen - und damit Skaleneffekte - haben. Dahingegen müssen sich Unternehmen, die an Range-Extender-Systemen arbeiten, auf einen sehr begrenzten Markt mit geringen Stückzahlen einstellen. Die Kunst wird sein, dennoch wirtschaftlich attraktive Angebote machen zu können und am Ende damit Geld zu verdienen.

Herzlichst,

Ihr

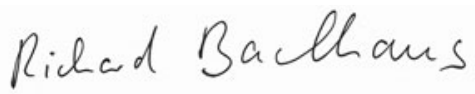

RICHARD BACKHAUS,

Stellvertretender Chefredakteur

Wiesbaden, 30. Januar 2014

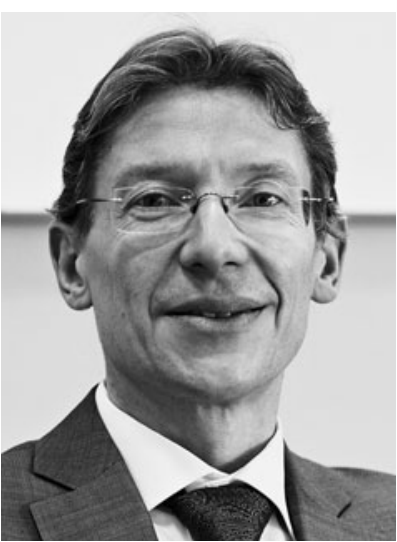

IAV - Ihr Partner

für Automotive

Engineering 